\title{
Scalable Agent-based Modelling with Cloud HPC Resources for Social Simulations
}

\author{
Peter Wittek \\ Swedish School of Library and Information Science \\ University of Borås \\ Borås, Sweden
}

\author{
Xavier Rubio-Campillo \\ Computer Applications in Science \& Engineering \\ Barcelona Supercomputing Centre \\ Barcelona, Spain
}

\begin{abstract}
New concepts like agent-based modelling are providing social scientists with new tools, more suited to their background than other simulation techniques. The success of this new trend will be strongly related to the existence of simulation tools capable of fulfilling the needs of these disciplines. Given the computational requirement of realistic agent-based models, high-performance computing infrastructure is often necessary to perform the calculations. At present, such resources are unlikely to be available to humanities researchers. Having developed Pandora, an open-source framework designed to create and execute large-scale social simulations in high-performance computing environments, this work presents an evaluation of the impact of cloud computing within this context. We find that the constraints of the cloud environment do not have a significant impact on the generic pattern of execution, providing a cost-effective solution for social scientists.

Index Terms-Cloud HPC Applications, High-Performance Computing, Agent-Based Modelling, Social Simulation, Humanities
\end{abstract}

\section{INTRODUCTION}

Computer modelling and simulations of complex systems have become dominant in scientific research over the last decade, providing important outcomes in biology, geology and life sciences, and also resulting in the birth of entirely new disciplines, such as bioinformatics, geoinformatics, health informatics, etc.

Recent years have seen a growing number of projects in Humanities and Social Sciences that use computer simulation as their main research tool. Agent-based models (ABMs) have been particularly successful in this regard due to their approach. An ABM defines the behaviour of any entity of a system that involves decision-making processes known as agents. These agents interact within a controlled environment that can be a real landscape with geographical features like vegetation, transport systems, etc., or an abstract domain. The researcher creates an initial scenario, giving values to certain parameters according to certain hypotheses that need to be validated. The simulation thus configured is executed in a series of regular time steps: an hour, a week, a year, or anything in between, depending on which time interval is appropriate for the experiment. For each of these time steps the agents evaluate the situation, plan which actions they want to execute and interact with each other and also with the environment. Finally, the results of several of these simulations are analysed in order to answer the questions defined at the beginning of the research.

A paradigmatic example of this methodology is SugarScape [14], a toy model specifically designed for social scientists. The authors built a virtual society from ground up using simple, individual behavioural rules. The results portray the emergence of several universal rules that are not explicitly identifiable in the initial model, but which are generated from the agent-agent and agent-environment interactions (such as migration, conflicts, trade, hierarchies, cultural identities, and so on) [11], [10]. This model has been so relevant that the structure of almost any ABM is inspired by it.

At a first glance, this approach is more interesting to Social Science and the Humanities than other techniques like formal mathematical models. The reason is that the generation of emergent properties that arose from the definition of individual agents can include both quantitative and qualitative concepts, combining behaviour aspects and data. Based on this bottomup approach, the explanation provided by an ABM is closer to how knowledge is acquired in social sciences. At the same time the formalization that a computer program requires can provide a higher level of rigour to the formulation and validation of hypotheses within these disciplines [10].

Despite these benefits, a researcher who attempts to use such a system should be cautious, because certain pitfalls and risks must be addressed in order to guarantee valid results and scientific quality. The most important point is that we should not accept the results of a simulation as directly applicable to reality. This problem is the result of misunderstanding the concept of modelling itself [6]. An excessive emphasis on individualism should also be avoided, a problem seen in badly designed agent-based models [22].

In a more practical sense, it is important that the models constructed are understandable and useful for humanities researchers and social scientists. If this is not the case, these models will not be published in journals of the discipline and their impact on the community of historians will remain insignificant however revealing the research is.

Finally, most of the existing ABM simulations tend to be small. Despite the fact that lots of emergent behaviours can be detected in these environments, certain phenomena can only be observed in large-scale scenarios. Moreover, the agents that are usually defined in Social Sciences are simple, being 
based on a set of predefined rules of actions. As ABMs are gaining a more widespread adoption, a need to introducing agents with more advanced behaviour is on the rise. Some models depends on agents with elaborated cognitive models and decision-making processes that avoid the need to hardcode every possible behaviour within the list of rules.

In order to confront these two issues, it is clear that future $\mathrm{ABM}$ will require further computational power. Only high-performance computing (HPC) resources are capable of dealing with large simulation scenarios containing agents with artificial intelligence algorithms and high computing costs. Until recently, these requirements were addressed by clusters and supercomputers. Given that these solutions are expensive to install, maintain, and operate, the use of large-scale ABM has been very limited. Cloud computing offers a new model of having access to infrastructure that is characterized by greater flexibility and better cost management, and is gaining use in high-performance computing [26].

With regard to social scientists, cloud HPC is certainly an enabler. The efforts of integrating HPC with cloud computing are ongoing [30], and humanities researchers are already discovering some potential applications [29]. Apart from largescale data analysis in the cloud [19], extending local resources with cloud infrastructure is also an attractive option [4]. In the long term, it should also be possible to replace batch scheduling with on-demand requests of simulation runs [15], and eventually bring simulation closer to real-time [27].

In this paper we discuss Pandora, an ABM framework designed for social scientists that uses HPC resources, and we look at the implications of replacing a traditional cluster with cloud resources. Since the simulation framework requires a high-speed interconnect, we expected low performance, but we witnessed only minor losses, proving that even a cheap cloud cluster can provide compelling computational power for empowering social simulation.

The rest of this paper is organized as follows. Section II describes our high-performance ABM framework. The issues related to $\mathrm{ABM}$ in a cloud environment are discussed in Section III. Section IV gives an in-depth account of our empirical experiments using cloud clusters in social simulation. Finally, Sections V and VI give some insight about future work and conclude the paper.

\section{The CHALlEnge OF PARALLEL AGENT-BASED SIMULATIONS}

The goal of distributing the workload of an ABM execution is a complicated task, due to the particularities of the techniques. The system is intrinsically communication intensive between the various components of the simulation. Agents need to gather knowledge from their environment, as well as from other agents in order to execute their decisionmaking processes. Once this phase is completed, there is a possibility that the agents will modify the environment and so will the other agents as well. These mechanics can be translated, in terms of parallelisation, to the need to share several layers of environmental data and agents; this process is quite convoluted. Furthermore, the execution of agent actions cannot usually be distributed within a single computer node (using threads or simple shared-memory parallelism like OpenMP), because there can be several conflicts related to agents accessing and modifying the same data at the same time. Finally, the scientific analysis of an ABM simulation will usually need to keep track of the evolution of the entire system from the beginning until the end of an execution. This means that the social scientist should have access to the state of the simulation at any given time step and at multiple levels of scale (from general statistics to the state of individual agents).

In order to address the dilemma between the potential of HPC in large-scale ABMs and the costs of communication, an approach based on the style of problem is necessary. Different problem types will allow for different solutions. For example, in some cases, the environment is simple or almost nonexistent, so agents can be dynamically assigned to computer nodes depending on the workload [17], while in other ones the interaction between agents can be almost nonexistent. In the present work, we focus on one of the most common type of ABMs, where agents interact within a spatially explicit environment. This environment is defined by several layers of information, that can be collected or modified by a set of agents located at a particular spatial position. These models are used in several applications, from archaeology [16] to urban dynamics [7]. It is worth noting that the space where agents interact does not have to model a realistic environment. It can be defined according to the requirements of the social scientist (for example, a knowledge space [9]).

Taking a closer look at these models, they have a list of common properties that can be used to distribute their execution. For example, in the case of disciplines that deal with past societies (e.g., archaeology, history) we should take into account the following properties:

- Importance of environment - Several social research disciplines are interested in the intrinsic relations between any society and the surrounding landscape where it exists. For this reason, ABMs designed to answer hypotheses about these sciences will need a substantial amount of spatial information, traditionally stored in a Geographical Information System [18]. These platforms are spatial databases where data are stored as vectors (database records with spatial coordinates) and raster maps (bidimensional matrices containing regularly distributed data). The agents' environment will be formed with this type of data, that can contain diverse information like digital elevation models, urban development plans, vegetation indexes, etc.

- Low density of agents - Past societies were not as populated as the ones we generally live in today. We will not need to reproduce situations with millions of agents, because in the vast majority of cases the models will contain at most tens of thousands.

- Intensive local communication - A world without telecommunications means that, within a small-to-mid scale, an agent will only interact with the environment 
and agents that are spatially close to it. These interactions can be intense, but an agent will not have to know the entire situation of the world; just the section of it within a given interaction range.

Pandora $^{1}$ is a novel open-source framework designed to accomplish a simulation environment corresponding to the above properties. It provides a $\mathrm{C}++$ environment that automatically splits the run of an ABM in different computer nodes. It allows the use of several CPUs in order to speed up the decision-making process of agents using costly AI algorithms. The package has also support for distributed execution and serialization through HDF5 [5], several analysis techniques (spatial analysis, statistics and geostatistics, etc.). In addition, pyPandora is a Python interface to the framework, designed to allow people with minimal programming background a tool to develop prototypes. The ability to develop ABMs using Python is an important feature for social scientists, because this programming language is also used in other common modelling tools, like Geographical Information Systems (i.e. QGIS [24] and ESRI ArcGIS ${ }^{2}$ ). PyPandora allows researchers to create ABMs using a language that they already know, and enables the design of a common framework where one can combine simulation, spatial analysis and geostatistics. Finally, the package is complemented by Cassandra, a visualization tool created to detect spatiotemporal patterns generated by the simulation. This application allows any user to load the data generated by a Pandora parallel execution into a single computer and analyse it.

The workflow of Pandora is defined in Figure 1; after defining the model that we would like to create, a first implementation can be written using Python scripting capabilities, and results will be analysed using several analysis tools. If there is a need for additional computing resources, a $\mathrm{C}++$ variant will be created. Pandora will automatically deal with the details of distributing the computational load of the simulation, so the researcher does not need to have any additional knowledge on parallel or distributed systems. Finally, the results of the distributed execution can be analysed as the same tools than the first prototype.

Pandora defines the environments where agents live as sets of layers containing raster map structures, following GIS standards. The world of the simulation is evenly divided among computer nodes, and each one of them will own a section of the environment, as well as the agents located in this part of the world. Data and agents located in the border between adjacent nodes will be copied and sent to neighbours at every time step, in order to keep data up-to-date in the entire scenario. The range of this border will be defined as the maximum interaction range of any agent, because this is the horizon of actions of any component of the simulation.

This approach solves the problem of distribution of workload for modelling historic societies, where advanced telecommunication systems were non-existent. The solution is highly

\footnotetext{
${ }^{1}$ https://github.com/xrubio/pandora

${ }^{2}$ http://www.esri.com/software/arcgis/index.html
}

scalable, given the fact that every computer node will need to communicate, at most, with 8 neighbouring nodes, independently of the total size of the simulation. Nevertheless, the issue of collision between agent's actions is still present. Pandora avoids these conflicts by splitting the section of every computer node in 4 equal parts, numbering 0 to 3 , as it can be seen in Figure 2. The agents contained in all 0 sections will be executed simultaneously without the possibility of conflicts, given the fact that they are not adjacent. Once all of them are finished, modified border data is sent to the neighbours, and a new section will begin its execution (1, 2 and finally 3 ). Finally the entire state of the simulation is serialized and a new time step can be evaluated.

Pandora parallelises this spatial distribution in different nodes using the MPI protocol. In addition, OpenMP is used to distribute computing workload of agents execution across different CPUs within a node. A common trend was detected while analysing different ABM platforms: the most intensive CPU tasks are the steps of gathering information and deciding which actions must be executed every time step. For this reason, the typical single-node method that an agent executes every time step $^{3}$ is split in three different methods. In the first one, updateKnowledge, an agent cannot modify the environment or the state of other agents; it only gathers information. In the second one, selectAction, the agent executes the decision-making process and chooses an action (it still cannot modify anything). Once every agent has chosen what it wants to do, Pandora executes the actions of the agents sequentially. Finally, the third method that the user must specify is updateState, where any agent can modify its internal state evaluating the results of its actions. This explore-decide-update cycle allows Pandora to distribute the execution among different CPU cores sharing the same memory space, as the first two steps (usually the most costly) can be automatically parallelised using OpenMP. The third one is executed sequentially, thus avoiding conflicts between actions and state updating.

At a first glance this structure could seem more complicated than just defining one method, but the division of an agent's execution in these three steps is more consistent than the traditional ABM approach. The reason is that the implementation using a single method mixes the different stages of a theoretical agent's cycle, that should be correctly specified while modelling the scenario, as can be seen in Figure 3. Dividing the execution using the three different steps avoids this problem, and allows the researcher to move from theory to implementation in a smoother way [25].

\section{AgEnT-BASED MODELLING IN THE CLOUD}

Tools like Pandora are solving current limitations of social simulation in terms of software applications (see also RepastHPC [3] and GridABM [12]), but there are issues related to needed infrastructure as well. The execution of a large-scale

\footnotetext{
${ }^{3}$ It is defined in every single common ABM platform like Netlogo [28](method tick), MASON [20](method step) and RePast [21](method step)
} 


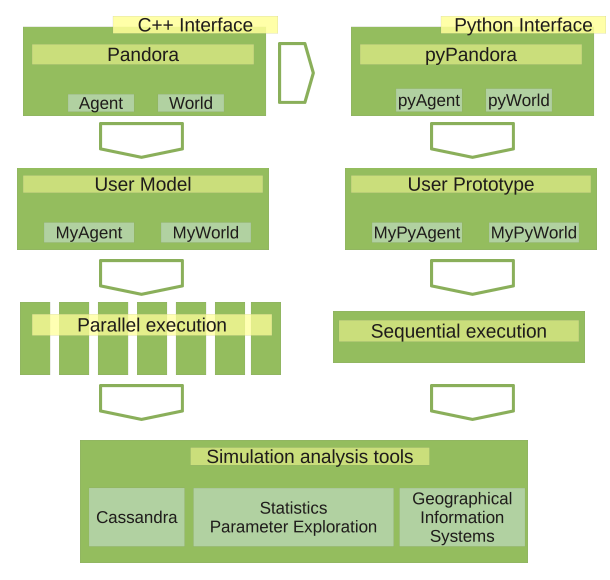

Fig. 1. Implementation workflow of an ABM using Pandora

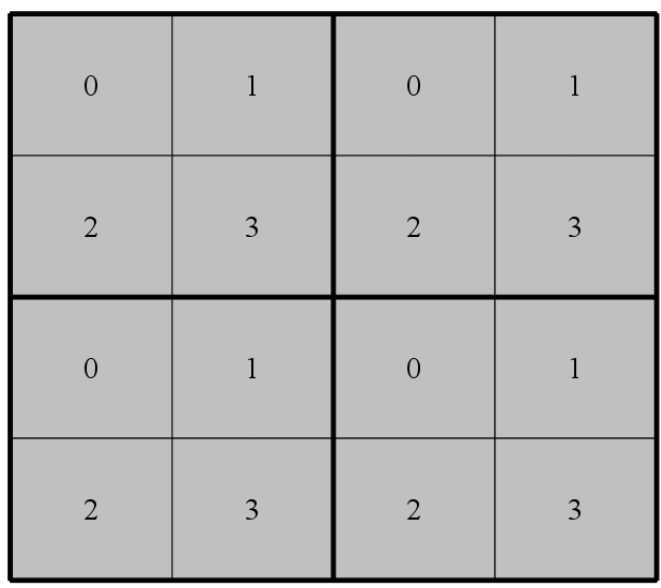

Fig. 2. The execution of any computer node is divided in 4 different sections, that are treated sequentially

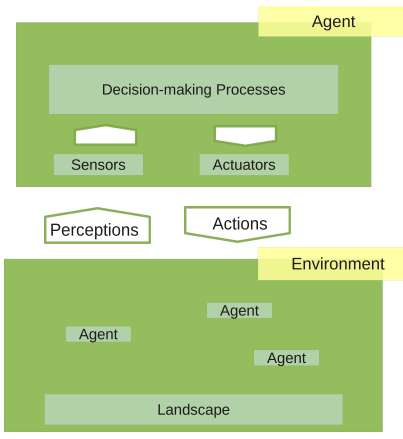

Fig. 3. The execution cycle of an agent

$\mathrm{ABM}$ involving numerous or complex agents will often require considerable computational power. Parallel and distributed
ABM asks for an HPC cluster to be able to efficiently test a number of hypotheses. Such resources are unlikely to be available to the researcher whose main interest is in using the results of the simulation.

It is also important to recognize the ad-hoc nature of these computations. Once the hypotheses are tested and data generated, visualization can be performed on an ordinary computer. Therefore it is hard to justify an investment in HPC resources, as they would idle most of the time. The associated maintenance cost is not negligible either. This is the fundamental reason for cloud HPC in ABM simulations.

The flexibility and cost-effectiveness of cloud computing infrastructure has already been recognized for HPC solutions. The underlying implementation differences and the different levels of abstraction cause problems, however. Using several applications that represent typical supercomputing centre workload, it has been indicated that a cloud cluster can be up to twenty times slower than a modern HPC system [13]. The type of interconnect of the cloud provider was sugested as the component that limits performance and creates significant variability.

Recognizing the importance of these issues, some cloud providers offer special cluster instances. These instances are different from standard instances not just in the interconnect, but also in the level of virtualization. Cluster cloud instances allow a tighter control over the hardware, not just abstracted computational cores. This kind of virtualization lets the guest virtual machine run as if it was on a native hardware platform with the exception that it still uses paravirtual network and storage drivers for improved performance.

\section{DISCUSSION}

We are interested in the evaluation of Cloud Computing for real case studies. For this reason, the tests executed an ABM developed inside SimulPast (http://www.simulpast.es), a project focused on integrating social simulation techniques within the stablished research methodology of the discipline. 
For these experiments we used the ABM developed for Case Study $1^{4}$. The simulation explores the competition between two different life strategies (hunter/gatherers and agropastoralists) within an arid environment with monsoonic climate. The general scenario is extremely detailed, modelling a zone of $40 \times 40$ kilometres using a resolution of $30 \mathrm{~m}$ (totalling $1600 \times 1600$, roughly 2.5 million cells) with several different layers portraying environmental information. Every run executed a simulation of a year, where biomass resources and agents' actions are updated daily. As we see, this is the type of model defined before, where the number of agents will not be huge, but every component is quite detailed (increasing the quantity of data to communicate between nodes, as well as CPU requirements).

The first benchmark that we designed wanted to understand the performance of the different systems for a computeintensive task. Each simulation would create an initial population of 100 agents. The decisions made for each agent were chosen using a Markov Decision Process (MDP) [2]. This is a CPU consuming algorithm that acts as a planner for the agent: given a set of goals, the agent will simulate on its own the accumulated effects of executing different available actions. Thus, every time step the MDP will explore potential benefits and costs of a large set of possible actions using a defined policy for a search depth defined by parameter. When the process is finished the agent will choose to execute the best action for the next state (where the algorithm will be executed again).

The second one was focused on communication intensive tasks. Using the same ABM, the decision making process of the agent was substituted by a simple programmed policy, cheap in terms of CPU but less efficient than the MDP. The initial population was four times larger (400 agents) to increase communication demands, and the amount of raster data to send and store was multiplied by 20: the interaction horizon was increased to 100 cells, and the number of stored raster maps went from 2 to 10 , setting the number of cells that need to be transmitted between adjacent computer nodes to 900.000 million per time step instead of 50.000) $)^{5}$.

The combinations of tests using these two benchmarks and different platforms allowed us to stress different aspects of the systems.

\section{A. Experimental configuration}

Our local cluster was the MareNostrum supercomputer at the Barcelona Supercomputing Centre. Each node has two dual-core IBM 64-bit PowerPC 970MP processors running at $2.3 \mathrm{GHz}$. It uses the Myrinet high-speed network for internode communication.

We relied on Amazon Web Services (AWS) for cloud instances. Having benchmarked the available instance types, we identified two types that suited our purposes. The m1.large

\footnotetext{
${ }^{4}$ http://simulpast.imf.csic.es/sp/index.php/wps/case-studies/cs1.html

${ }^{5} \mathrm{An}$ ODD description of this ABM, its source code and the different configurations, can be downloaded from https://github.com/xrubio/pandora/ tree/master/pandora/examples/gujarat/doc/
}

instances come with two virtual cores with 2 EC2 Compute Units each. The EC2 Compute Unit is an abstraction that indicates the approximate CPU performance of the instance. We found the performance very similar to the PowerPC cores in our local configuration, hence we expected that the differences in the interconnect quality would show distinctly. Choices were more limited when it came to special cluster instances. The smallest instance type, cc1.4xlarge, offers two quad-core Intel Xeon X5570 CPUs with a Gigabit Ethernet connection. This is several magnitudes faster than the PowerPC CPUs in MareNostrum. We ran two sets of experiments on this instance type: unthrottled and throttled. The throttled one reduced the number of maximum cores used to two, resulting in comparable computational power.

Due to a current limitation in the ABM library, only Cartesian grids of equal sides are suitable for execution. We tested cluster sizes of $2 \times 2$ and $3 \times 3$.

During the execution of benchmarks we collected the traces with Extrae [8] and analysed the results with Paraver [23]. Since we were chiefly interested in the impact of the infrastructure on interprocess communication, we looked at two key indicators:

- The average length the processes spend on communication. This indicator describes parallel efficiency.

- The average/maximum ratio, where maximum is the longest time any process spends on computation. This indicator describes the load balance, it is related to the variation in computational time across the processes.

We also looked at the communication pattern by plotting the traces.

\section{B. Computational performance}

1) Compute-intensive task: Figures 4 and 5 show the average and average/maximum indicators, respectively. The load balance is nearly identical, and the average improves if the cloud cluster instance is unthrottled. This is not surprising, given that the communication was limited across the instances. The same is confirmed by the actual traces (Figure 6), the overall communication pattern is similar across the different infrastructures, although the regular and throttled cloud instances spend a considerably longer time in MPI_Send.

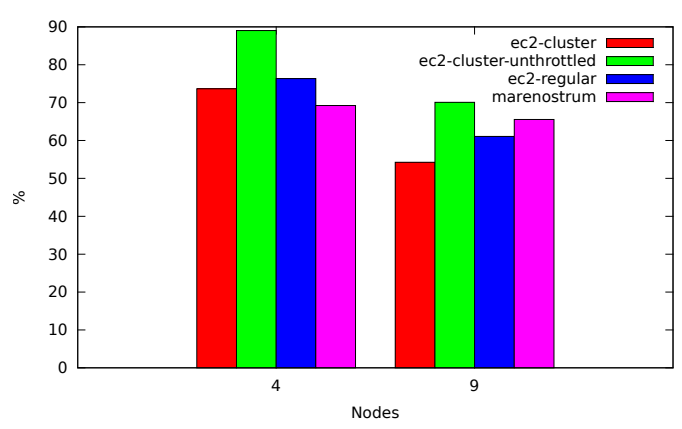

Fig. 4. Average load, compute-intensive task 


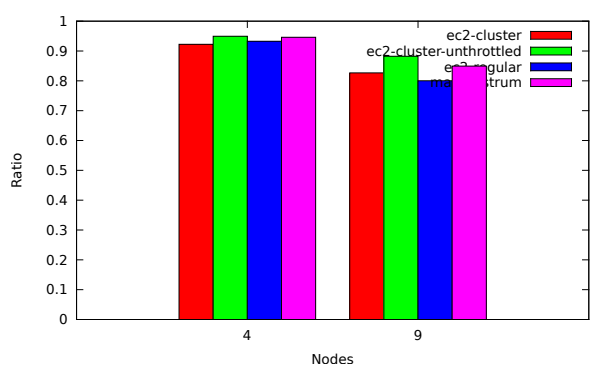

Fig. 5. Average/maximum, compute-intensive task

2) Communication-intensive task: Average load changes dramatically with regular cluster instances in the communication-intensive scenario, dropping from close to $80 \%$ to below $50 \%$ (Figure 7). The role of the interconnect is apparent.

The variation in load balance is far more even (Figure 8). This leads us to conclude that an effort is made by the cloud provider to place simultaneously launched virtual instances on physically nearby computers even in the case of standard instances. Otherwise we would witness a more haphazard pattern.

The differences in the traces on four nodes are striking (Figure 9). The overall pattern is similar between the local cluster and the unthrottled cloud instance, with the exception of the increased time in MPI_Send which we also observed in the compute-intensive case. In the other two cases, the time spent on MPI communication dramatically increases, even with the Gigabit Ethernet connection. The increase comes from nonasynchronous MPI calls, which explains while the throttled cluster instance shows a very different communication pattern from the unthrottled variant. A combination of the low CPU performance and slower interconnect results in the somewhat unexpected results.

\section{Cost}

At the time of writing, a regular m1.large instance costs $\$ 0.32$ an hour on demand, whereas the cluster cc1.4xlarge instance is $\$ 1.3$. While communication performance is certainly better on the latter type, we believe the difference is probably not worth four times the cost. ABM is typically not a memorybound or I/O-bound application, a larger number of relatively simple nodes work better, although communication overhead will get worse as the cluster size increases.

\section{FUTURE WORK}

Cloud instances with high-performance graphics processing units (GPU) are becoming available. While ABM is not straightforward to adapt to the GPU-programming paradigm, considerable speedups have been demonstrated [1]. Using GPU-based cloud instances is more expensive if we look at the price of the instance hour, but it is also likely that a smaller cluster will finish the work in a shorter stretch of time. We would like to pursue the investigation further in this direction.

\section{CONCLUSION}

Social scientists are unlikely users of HPC applications and the infrastructure-as-a-service aspect of cloud computing is also typically targeted on other application domains. Yet, given the expected increase of agent-based modelling in social sciences, we believe we identified a potential candidate for truly meaningful cloud-based HPC. The key contributions of this paper are as follows:

- Demonstrating the need for cloud HPC in social simulations for humanities researchers.

- Deploying a high-performance agent-based modelling framework in a cloud environment.

- In-depth performance comparison of agent-based simulation in a traditional cluster and in cloud clusters.

Results of this work show that social scientists interested in developing large-scale ABMs are currently able to exploit both the capabilities of supercomputers and the flexibility of cloud computing infrastructure. The choice will depend on budget, but also on the properties of the case studies that require modelling. Irrespective of the constraints, the possibility of choosing different HPC systems to efficiently execute parallel ABMs provides the next step towards the generalization of HPC resources in social science disciplines.

\section{ACKNOWLEDGEMENT}

This work was carried out while the first author was visiting the Department of Computer Applications in Science \& Engineering at the Barcelona Supercomputing Center, funded by the "Access to BSC Facilities" project of the HPC-Europe2 programme (contract no. 228398). The second author is supported by the SimulPast Project (CSD2010-00034), funded by the CONSOLIDER-INGENIO2010 program of the Ministry of Science and Innovation - Spain. The authors also thank the help of the SimulPast team involved in the development of the model.

\section{REFERENCES}

[1] B. Aaby, K. Perumalla, and S. Seal, "Efficient simulation of agentbased models on multi-GPU and multi-core clusters," in Proceedings of SIMUTOOLs-10, 3rd International ICST Conference on Simulation Tools and Techniques, Malaga, Spain, March 2010.

[2] B. Bonet and H. Geffner, "Action selection for MDPs: Anytime AO* vs. UCT," in Proceedings of AAAI-12, 26th Conference on Artificial Intelligence, Toronto, Canada, July 2012.

[3] N. Collier and M. North, "Repast HPC: A platform for large-scale agentbased modeling," in Large-Scale Computing Techniques for Complex System Simulations, W. Dubitzky, K. Kurowski, and B. Schott, Eds. Wiley, 2011, pp. 81-110.

[4] M. De Assunção, A. Di Costanzo, and R. Buyya, "Evaluating the costbenefit of using cloud computing to extend the capacity of clusters," in Proceedings of HPDC-09, 18th ACM International Symposium on High Performance Distributed Computing, Garching, Germany, June 2009, pp. 141-150.

[5] M. Folk, A. Cheng, and K. Yates, "HDF5: A file format and I/O library for high performance computing applications," in Proceedings of SC-1999, 12th Conference on Supercomputing, Portland, OR, USA, November 1999.

[6] V. Gaffney and G. Bradley, "Who's in command here? the digital basis of historical, military logistics," in General Issues in the Study of Medieval Logistics: Sources, Problems and Methodologies, J. Haldon, Ed. Brill Academic Publisher, 2006, pp. 37-69. 


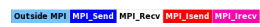

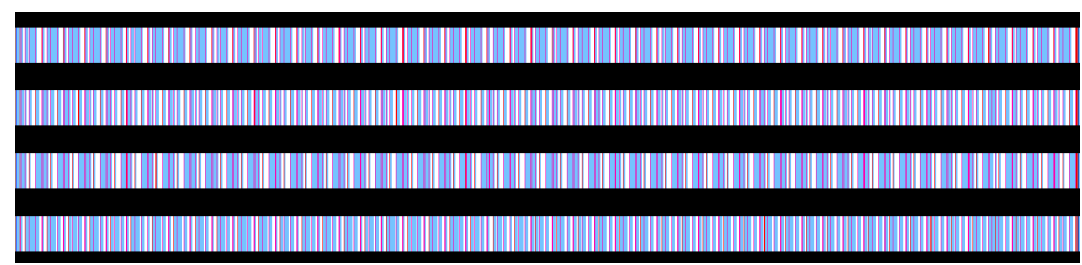

(a) MareNostrum

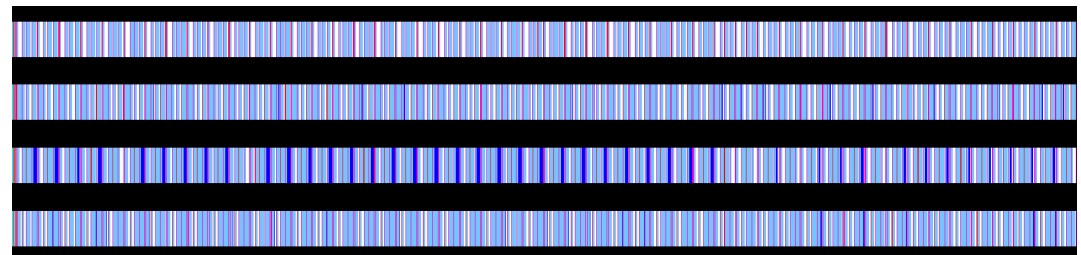

(b) EC2 Regular

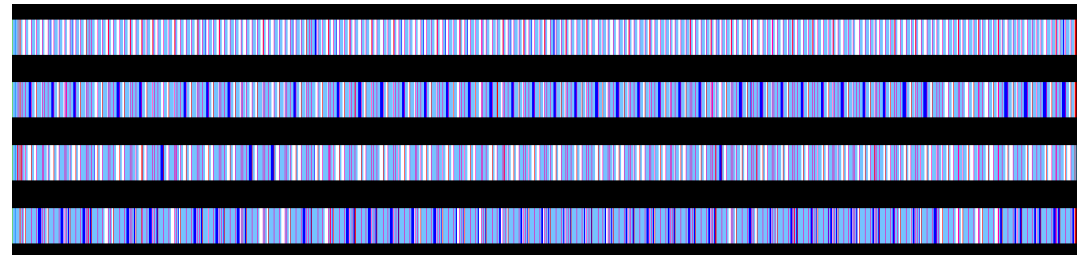

(c) EC2 Cluster Throttled

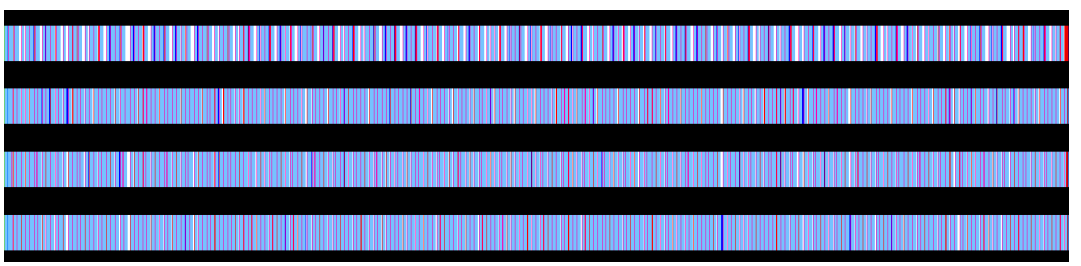

(d) EC2 Cluster Unthrottled

Fig. 6. MPI trace timeline on four nodes, compute-intensive task

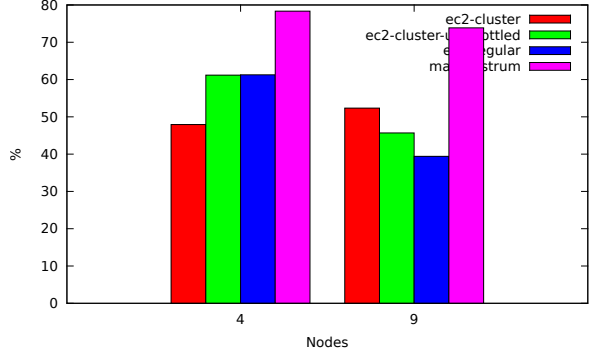

Fig. 7. Average load, communication-intensive task

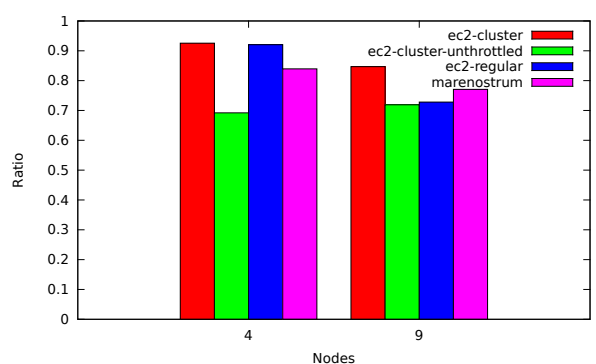

Fig. 8. Average/maximum, communication-intensive task
[7] J. Galan, A. Lpez-Paredes, and R. del Olmo, "An agent-based model for domestic water management in valladolid metropolitan area," Water Resources Research, vol. 45, p. 17, 2009.

[8] H. S. Gelabert and G. L. Sánchez, "Extrae: Users' guide manual," Barcelona Supercomputing Centre, February 2012.

[9] N. Gilbert, "A simulation of the structure of academic science," Sociological Research Online, vol. 2, no. 2, p. 17, 1997.

[10] - Agent-Based Models. SAGE Publications, 2008.

[11] N. Gilbert and K. Troitzsch, Simulation for the Social Scientist. Open University Press, 2008.

[12] L. Gulyás, A. Szabó, R. Legéndi, T. Máhr, R. Bocsi, and G. Kampis, "Tools for large scale (distributed) agent-based computational experiments," in Proceedings of CSSSA-11, Computational Social Science
Society of America Annual Conference, Santa Fe, NM, USA, October 2011.

[13] K. Jackson, L. Ramakrishnan, K. Muriki, S. Canon, S. Cholia, J. Shalf, H. Wasserman, and N. Wright, "Performance analysis of high performance computing applications on the Amazon Web Services cloud," in Proceedings of CloudCom-10, 2nd IEEE International Conference on Cloud Computing Technology and Science, Indianapolis, IN, USA, December 2010, pp. 159-168.

[14] E. J.M. and A. R.L., Growing Artificial Societies: Social Sciences from the Bottom Up. MIT Press, 1996.

[15] G. Juve, E. Deelman, K. Vahi, G. Mehta, B. Berriman, B. Berman, and P. Maechling, "Scientific workflow applications on Amazon EC2," in Proceedings of eScience-09, 5th IEEE International Conference on 


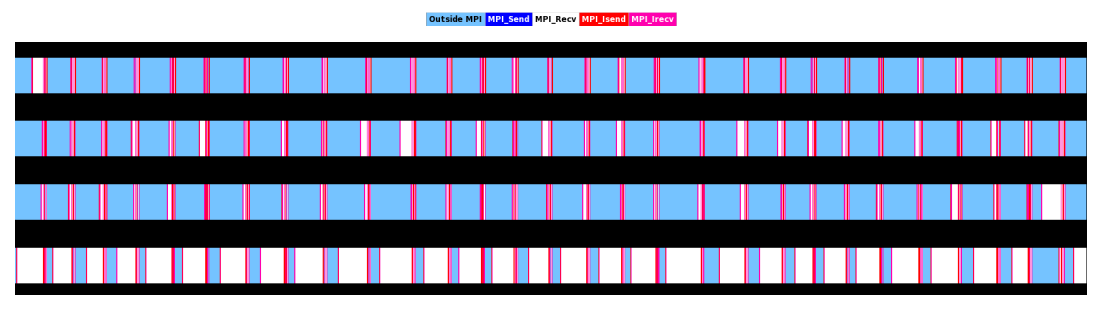

(a) MareNostrum

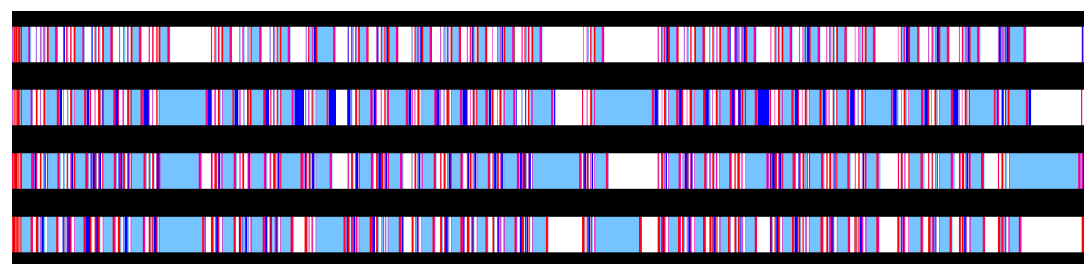

(b) EC2 Regular

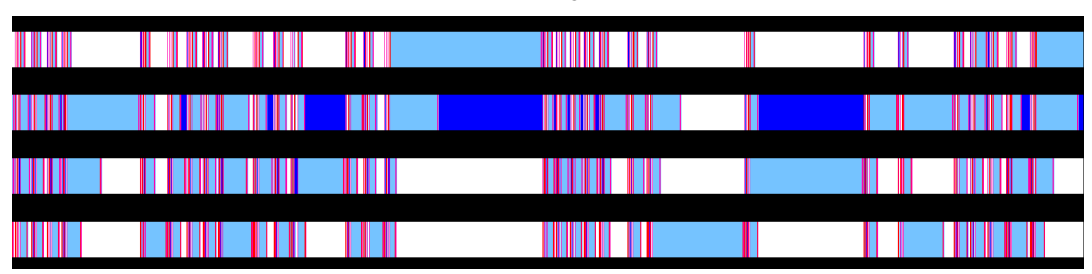

(c) EC2 Cluster Throttled

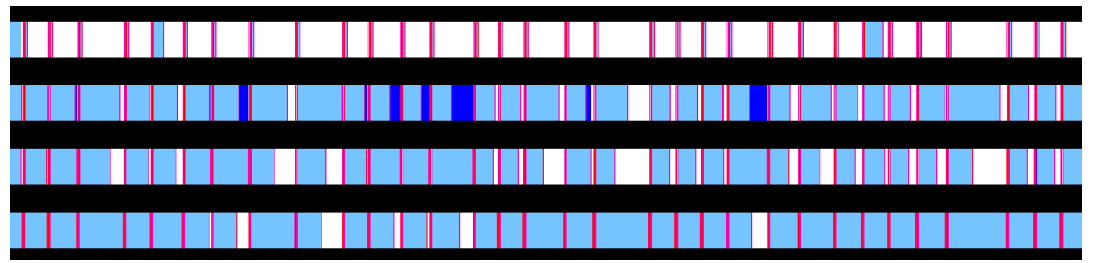

(d) EC2 Cluster Unthrottled

Fig. 9. MPI trace timeline on four nodes, communication-intensive task

E-Science Workshops, Oxford, United Kingdom, December 2009, pp. 59-66.

[16] T. Kohler and S. A. van der Leeuw, The Model-based Archaeology of Socionatural Systems. School for Advanced Research Press, 2007.

[17] Q. Long, J. Lin, and Z. Sun, "Agent scheduling model for adaptive dynamic load balancing in agent-based distributed simulations," Simulation Modelling Practice and Theory, vol. 19, pp. 1021-1034, 2011.

[18] P. Longley, M. Goodchild, D. Maguire, and D. Rhind, Geographic Information Systems and Science. John Wiley and Sons, 2001.

[19] S. Lu, R. Li, W. Tjhi, K. Lee, L. Wang, X. Li, and D. Ma, "A framework for cloud-based large-scale data analytics and visualization: Case study on multiscale climate data," in Proceedings of HPCCloud-11, Workshop on Integration and Application of Cloud Computing to High Performance Computing. Athens, Greece: IEEE, December 2011, pp. 612-617.

[20] S. Luke, Multiagent Simulation and the MASON Library, George Mason University, August 2011.

[21] M. North, T. Howe, N. Collier, and J. Vos, "A declarative model assembly infrastructure for verification and validation," in Advancing Social Simulation: The First World Congress, S. Takahashi, D. Sallach, and J. Rouchier, Eds., 2007.

[22] D. O'Sullivan and M. Haklay, "Agent-based models and individualism: is the world agent-based?" Environment and Planning, vol. 32, no. 8, pp. 1409-1425, 2000.

[23] V. Pillet, J. Labarta, T. Cortes, and S. Girona, "Paraver: A tool to visualize and analyze parallel code," Proceedings of WoTUG-18, 18th World OCCAM and Transputer User Group Technical Meeting, pp. 1731, April 1995.

[24] Quantum GIS Development Team, Quantum GIS Geographic
Information System, Open Source Geospatial Foundation, 2009. [Online]. Available: http://qgis.osgeo.org

[25] S. Russell and P. Norvig, "Intelligent agents," in Artificial Intelligence: A Modern Approach. Prentice Hall, 2012, pp. 34-63.

[26] C. Vecchiola, S. Pandey, and R. Buyya, "High-performance cloud computing: A view of scientific applications," in Proceedings of ISPAN09, 10th International Symposium on Pervasive Systems, Algorithms, and Networks, Kaohsiung, Taiwan, December 2009, pp. 4-16.

[27] H. Wang, Y. Ma, G. Pratx, and L. Xing, "Toward real-time Monte Carlo simulation using a commercial cloud computing infrastructure," Physics in Medicine and Biology, vol. 56, pp. N175-N181, 2011.

[28] U. Wilensky, NetLogo, Center for Connected Learning and ComputerBased Modeling, Northwestern University, 1999. [Online]. Available: http://ccl.northwestern.edu/netlogo

[29] P. Wittek and S. Darányi, "Leveraging on high-performance computing and cloud technologies in digital libraries: A case study," in Proceedings of HPCCloud-11, Workshop on Integration and Application of Cloud Computing to High Performance Computing, Athens, Greece, December 2011, pp. 606-611.

[30] T. Wlodarczyk and C. Rong, "An initial survey on integration and application of cloud computing to high performance computing," in Proceedings of HPCCloud-11, Workshop on Integration and Application of Cloud Computing to High Performance Computing, Athens, Greece, December 2011, pp. 612-617. 